\title{
Masking produced by spectral uncertainty with multicomponent maskers
}

\author{
DONNA L. NEFF and DAVID M. GREEN \\ Harvard University, Cambridge, Massachusetts
}

\begin{abstract}
The first experiment examined thresholds for signals at 250, 1000, and $4000 \mathrm{~Hz}$ presented simultaneously with maskers that had from 1 to 100 sinusoidal components. The number of masker components remained constant throughout a block of trials, but the component frequencies were drawn at random from a $5000-\mathrm{Hz}$ range for each presentation. Maskers with as few as 10 components produced more masking than maskers with up to 100 components or than broadband noise. The amount of masking decreased dramatically if the same masker occurred in both intervals of a forced-choice trial, even if the masker changed between trials. The second experiment examined whether judgments of the number of components present in a stimulus might aid signal detection. Such a strategy would not be effective, since about $50 \%$ more components are needed to discriminate between stimuli, regardless of the initial number of components. These experiments indicate that listeners sometimes are unable to use a single-filter detection strategy, even when it is advantageous to do so.
\end{abstract}

Ever since Fletcher's (1940) classic study of the masking effects of noise of different bandwidths, considerable evidence has accumulated that the peripheral auditory system can be modeled successfully as a bank of overlapping bandpass filters (see Scharf, 1970, for a review). Listeners are thought to detect a sinusoidal signal of known and fixed frequency by using an "auditory filter" centered at or near the signal frequency. Information outside this filter is thought to have relatively little effect, either positive or negative. In recent years, however, a number of investigators have demonstrated phenomena for which this classical energy-detection model appears inadequate. In particular, there are situations in which listeners appear to take advantage of energy in spectral regions remote from the signal frequency to improve performance, as in "profile analysis" (e.g., Green, 1983; Green \& Kidd, 1983; Green, Mason, \& Kidd, 1984; Spiegel \& Green, 1982) and "comodulation masking release" (e.g., Hall, 1985; Hall, Haggard, \& Fernandes, 1984). Perhaps it is not surprising that a sophisticated detector can do this if required. In this paper, we will present evidence of the opposite kind, evidence that there are masking conditions in which listeners are apparently unable to ignore spectral information far from the signal frequency, even when

This work was supported in part by the National Institutes of Health. Donna L. Neff was supported by a postdoctoral fellowship from NINCDS and a Biomedical Research Support Grant to Boys Town National Institute. We would like to thank Thomas E. Hanna for discussions of the conditions tested, Christine R. Mason for data collection for the second experiment, and Brian Callaghan and Theresa Langer for help with figure generation. Walt Jesteadt, Robert Shannon, and Patricia Stelmachowicz provided helpful comments and suggestions on this manuscript. Requests for reprints should be sent to Donna L. Neff, Boys Town National Institute for Communication Disorders in Children, 555 North 30th Street, Omaha, NE 68131. The present address for David M. Green is the Department of Psychology, University of Florida, Gainesville, FL 32611. it is clearly advantageous for them to do so. The implication is that they are unable to monitor the output of a single auditory filter, even though the signal is fixed in frequency.

Simultaneous masking experiments, in which the signal and masker are presented together in time, have long been used as a tool for investigating auditory processing. Numerous studies have measured the threshold for a signal in the presence of sinusoidal maskers or noise maskers ranging from narrow to broad bandwidths. Despite the obvious phenomenological differences between pure tones and noise, both are alleged to produce masking by exciting the peripheral channels so that stimulation by the signal is either blocked or rendered less effective. Differences in the masking effectiveness of tones and noise are attributed to differences in spectral content or envelope characteristics and to the filtering properties of the peripheral auditory system.

There is a continuum between these two types of maskers in that one can simulate a noise masker by adding together a sufficient number of pure tones, and several investigators have used this approach (e.g., Margolis, Dubno, \& Hunt, 1981; Neff \& Jesteadt, 1983; Schafer, Gales, Shewmaker, \& Thomson, 1950; Weber, 1984; see Papoulis, 1965, for a commonly used algorithm). For all these simulated noises, care was taken to use enough tones so that the tonal character of the individual components in the masker was replaced by the atonal, diffuse character that is associated with noise. There is little data on the number and spacing of tones necessary to reproduce the percept of broadband noise or on the relationship between the degree of atonality or "noisiness" of a stimulus and the masking it produces. Informal observations by Richards (1979) suggest that the quality of a broadband noise is reproduced by tones spaced $1 / 6$ octave apart. Schafer et al. (1950) found that a spectral density of 1 
sinusoid per hertz was necessary to produce the same masking as a broadband noise; this synthesized stimulus also was perceptually indistinguishable from analog noise. A recent paper by Hartmann, McAdams, Gerzso, and Boulez (1986) on the discrimination of spectral density found that listeners could not distinguish densities greater than 80 components per $1000 \mathrm{~Hz}$. As noted by the authors, this is considerably less than the 1,000 components per $1000 \mathrm{~Hz}$ reported to produce equivalent masking by Schafer et al. (1950).

In addition to the issue of recreating broadband noise with some minimum, but probably rather large, number of components, there is also the issue of the properties of maskers with few components. Early studies of the ability of listeners to resolve individual components (Pollack, 1964) or to judge the number of tones presented simultaneously (Thurlow \& Rawlings, 1959) showed surprisingly poor performance for complex spectra with no more than four tones. The methods of stimulus generation and presentation available at that time probably contributed to the listeners' difficulty, but the results are intriguing nonetheless. Corresponding masking data were not collected.

It is important to note, particularly in relation to the present study, that masking can be produced by factors other than energy's falling within a limited band around the signal frequency. With sequential tonal patterns, it has been shown that uncertainty in the context in which a target tone is presented can significantly degrade performance (e.g., Watson, 1976; Watson \& Kelly, 1981; Watson, Kelly, \& Wroton, 1976). Watson has termed this phenomenon "informational masking" to distinguish it from masking produced by energy at the signal frequency. Similar, although typically smaller, effects of stimulus uncertainty have been observed in simultaneous masking when the signal is fixed in frequency but the masker (either a noise or a tonal complex) is changed from trial to trial (Spiegel \& Green, 1982; Spiegel, Picardi, \& Green, 1981).

In the present study, our intent was to vary the number of tones in a multicomponent simultaneous masker to determine the minimum number necessary to produce stimuli with the same masking properties as broadband noise, based on corresponding experiments with sinusoidal gratings in vision (e.g., Richards, 1979). The initial results, however, were quite different from our original expectations in that very small numbers of components produced large amounts of masking. For these conditions, the tonal quality of the masker was often very evident. Unlike the majority of previous studies of simultaneous masking that used sinusoids or combinations of sinusoids, the spectral content of the masker was changed with every stimulus presentation, as it would if the masker were random noise. Also, unlike Hartmann et al. (1986) or Schafer et al. (1950), we purposely did not distribute our components uniformly across the frequency range. The combination of these factors produces appreciable masking that is not easily explained by peripheral theories that depend upon energy's falling within a limited band around the signal or upon the maskers' inherent statistical variation.

The two experiments presented below used essentially the same stimuli, but addressed different questions. The first experiment examined the masking produced by multicomponent maskers on signals at several different frequencies under conditions of high spectral uncertainty. The second experiment examined the ability of the listeners to use judgments of the number of components present in a stimulus to aid signal detection.

\section{EXPERIMENT 1}

\section{Method}

Subjects. Three normal-hearing listeners (thresholds below $10 \mathrm{~dB}$ $\mathrm{HL}$ at audiometric frequencies from 250 to $4000 \mathrm{~Hz}$ ) were paid for their participation. One of the listeners had extensive experience in psychoacoustic masking tasks; all listeners were given at least $10 \mathrm{~h}$ practice before data collection began. They listened binaurally in individual IAC chambers through TDH-50 headphones. The experimental sessions usually consisted of 10 to 12100 -trial runs, completed in blocks of three or four runs separated by rest periods.

Stimuli and Procedure. With the exception of broadband noise, the stimuli were computer generated, played through 16-bit digitalto-analog converters at a rate of 12,500 points/sec, and low-pass filtered at $6250 \mathrm{~Hz}$. All stimuli were square-gated with a laboratorybuilt electronic switch. Stimulus presentation, timing, and response recording were computer controlled.

A two-alternative, forced-choice (2AFC), adaptive procedure was used to determine threshold for the signal in quiet and in the presence of a masker, with a decision rule that estimated the $70.7 \%$ correct point on the psychometric function (Levitt, 1971). An initial step size of $4 \mathrm{~dB}$ was reduced to $2 \mathrm{~dB}$ on the fourth reversal. Threshold was defined as the average of the reversal levels recorded during each 100-trial run beginning with the fourth reversal. The results, of at least eight 100-trial runs were averaged for each listener and the mean threshold and standard deviation across listeners was determined.

Lights were used to demark the listening intervals and provide correct-answer feedback. Each interval contained either a 200-msec masker or the masker plus a 200-msec sinusoidal signal. Signals at 250,1000 , and $4000 \mathrm{~Hz}$ were used, with signal frequency fixed for at least three consecutive 100-trial runs. There were two types of maskers. One, which served primarily as a reference condition, was an analog broadband noise, low-pass filtered at $5000 \mathrm{~Hz}$ and presented at 60-dB SPL total power. The other was a multicomponent sinusoidal complex. The number of sinusoidal components in the complex was varied from 1 to 100 . Component frequencies were drawn at random from the same frequency range as the broadband noise $(0-5000 \mathrm{~Hz})$. To ensure that the components were orthogonal, the minimum allowable spacing between component frequencies was $5 \mathrm{~Hz}$, given the 200-msec stimuli. Thus there were no masker components within a $10-\mathrm{Hz}$ interval around the signal. The signal frequency could not be drawn as a masker component. To give the components the properties each would have if excised from noise, the phase of each component was random, drawn from a rectangular distribution, and the amplitude of each component was also random, drawn from a Rayleigh distribution. Although the number of components in the maskers varied across conditions, average total power was held constant at $60 \mathrm{~dB}$ SPL.

For each condition, 50 different masker waveforms were generated with the specified number of components. In a block of 100 
trials, a different masker waveform was drawn at random from the 50 available for each interval of each trial. Signal frequency and the number of components in the maskers remained constant across trials.

\section{Results and Discussion}

Results for the lowest signal frequency, $250 \mathrm{~Hz}$, are shown in Figure 1 in terms of the amount of masking (masked threshold minus quiet threshold in decibels) as a function of the number of tones in the masker complex. A logarithmic scale is used for the abscissa simply to allow performance for maskers with less than 10 components to be seen more clearly. The dashed line indicates that $10.0 \mathrm{~dB}$ of masking (standard deviation $=2.9 \mathrm{~dB}$ ) is produced at this signal frequency by the broadband noise. ${ }^{1}$ For small numbers of components, increasing the number of components leads to more masking. The masking produced at $250 \mathrm{~Hz}$ when only 5 components fall in the $5000-\mathrm{Hz}$ range is equal to that produced by the broadband noise. Adding more components produces up to $10 \mathrm{~dB}$ more masking than that produced by the noise. There is a broad peak for maskers with 20 or 50 components, followed by a plateau or slight decline. The amount of masking does not return to that produced by broadband noise for 100-component maskers, the maximum number tested.

The results are more dramatic for the $1000-\mathrm{Hz}$ signal shown in Figure 2. In this case, the broadband noise produces $36.4 \mathrm{~dB}$ of masking (standard deviation = $4.2 \mathrm{~dB}$ ). What is immediately apparent is the large increase in masking as the number of components is increased from 1 to 10 . Two components, falling at ran-

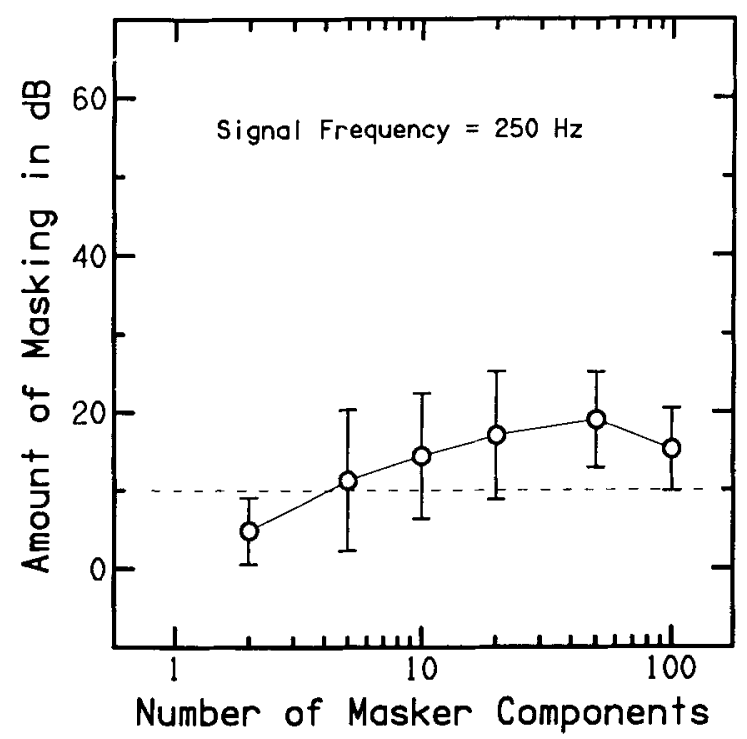

Figure 1. Amount of masking in decibels as a function of the number of sinusoidal components in the masker for a signal frequency of $250 \mathrm{~Hz}$. The data are averages and standard deviations across three listeners. The dashed horizontal line indicates the amount of masking produced by a broadband noise at the signal frequency. Each point is based on 8 to 10 100-trial runs per listener. Average quiet threshold at $250 \mathrm{~Hz}$ was $29.5 \mathrm{~dB}$ SPL.

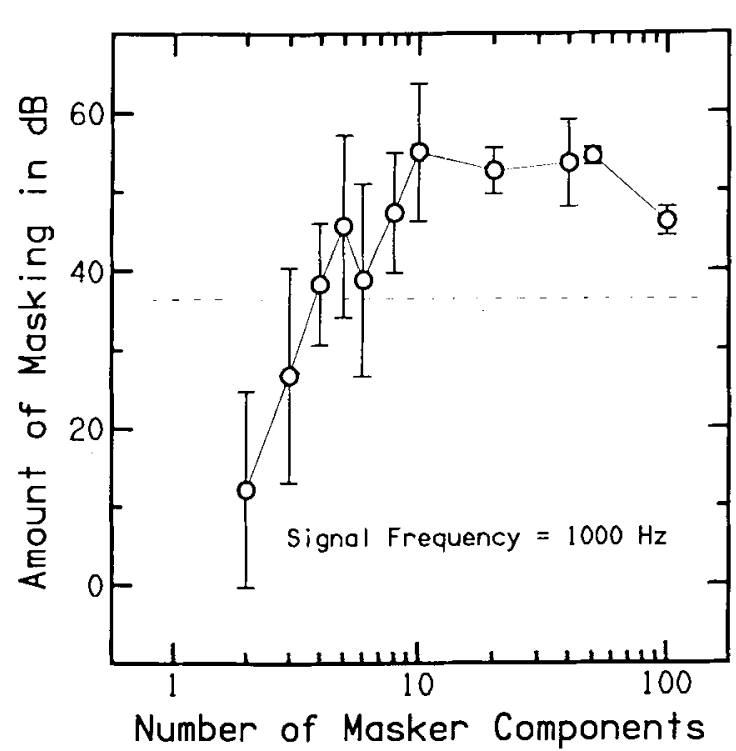

Figure 2. Same as Figure 1 except for a signal frequency of $1000 \mathrm{~Hz}$. Average quiet threshold at $1000 \mathrm{~Hz}$ was $3.8 \mathrm{~dB}$ SPL.

dom in the $5000-\mathrm{Hz}$ range, can produce $10 \mathrm{~dB}$ of masking. Three or 4 components produce the same amount of masking as the broadband noise; 10 components produce 10 to $20 \mathrm{~dB}$ more masking than the noise. The amount of masking remains essentially unchanged as the number of components is increased from 10 to 50 , but declines for 100 components. As indicated by the error bars, there is large variability in performance across listeners for maskers with few components and relatively stable performance for larger numbers of components. This same pattern occurs across repeated conditions for each listener as well. The variation in energy across the different masker samples, which is larger for maskers with small numbers of components, is probably a major factor.

Results for the 4000-Hz signal, shown in Figure 3, are almost identical to those observed for the $1000-\mathrm{Hz}$ signal. The broadband noise produces $38.5 \mathrm{~dB}$ of masking (standard deviation $=4.1 \mathrm{~dB}$ ) at $4000 \mathrm{~Hz}$. In this case, one masker component produces over $20 \mathrm{~dB}$ of masking and two components produce over $40 \mathrm{~dB}$ of masking. Again, there is a steady increase in masking for maskers with up to 10 components, followed by a gradual decrease in masking that approaches but does not reach that for broadband noise for 100-component maskers.

Regardless of signal frequency, the masking observed is probably a combination of informational masking produced by some aspect of stimulus uncertainty and energetic masking produced by masker energy's falling within the auditory filter around the signal. The fact that the functions for 1000 - and $4000-\mathrm{Hz}$ signals are very similar, particularly for maskers with 10 or more components, suggests that this is not a form of masking that depends predominantly on the size of the critical band or some other estimate of the auditory filter, all of which differ significantly across frequency. Overall, far less masking 


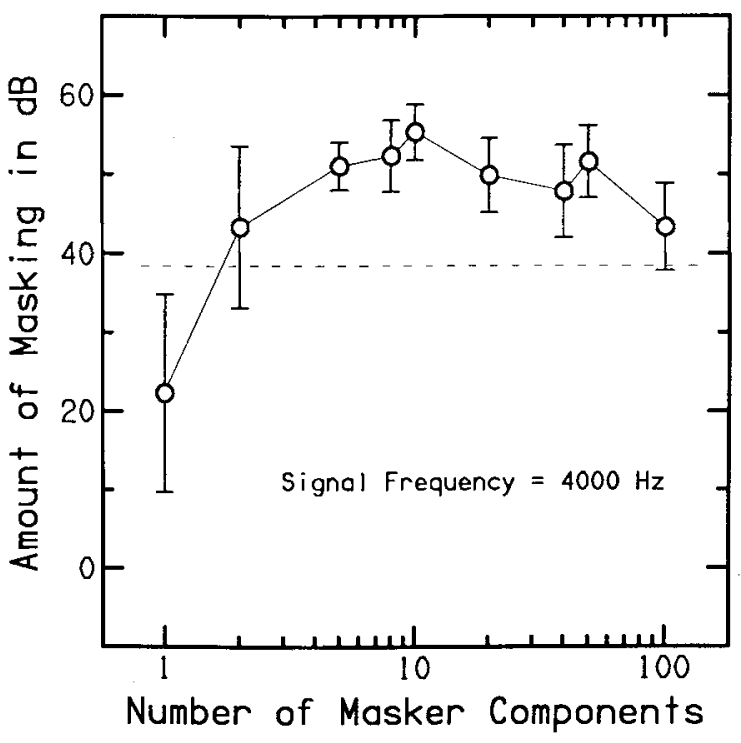

Figure 3. Same as Figure 1 except for a signal frequency of $4000 \mathrm{~Hz}$. Average quiet threshold at $4000 \mathrm{~Hz}$ was 9.6 dB SPL.

is produced in all conditions for the $250-\mathrm{Hz}$ signal. Most of this difference, however, is due to the use of amount of masking rather than masked threshold to characterize the data, inasmuch as quiet thresholds at $250 \mathrm{~Hz}$ are 20-26 dB higher than at the other two signal frequencies. It is also possible that the lower amounts of masking for the 250-Hz signal might reflect some sort of anchor effect in that few masker components will fall below that signal frequency, whereas masker components are more likely to fall on both sides of the higher signal frequencies.

Another argument in support of a substantial contribution of informational masking to these data is that, particularly for maskers with small numbers of components, the frequency range from which components were drawn was large enough for few components to have fallen within the signal's filter. As a simplistic example, let us assume a rectangular critical band filter at $1000 \mathrm{~Hz}$, for which a generous estimate of the bandwidth is $160 \mathrm{~Hz}$ (Scharf, 1970). Given the 5000-Hz frequency range from which masker components were drawn, the probability of any given component's falling within that band is $160 / 5,000$, or about $3 \%$. For five components, the probability is still only $15 \%$. Furthermore, as the number of components increases, the average power of individual components decreases because the overall power of the masker was held constant. Thus, until the number of masker components is very large, masking produced by energy within a critical band around the signal is not very great. Our data suggest that even 100 components are not sufficient to simulate the effects of broadband noise. Instead, the change in the quality of the maskers from presentation to presentation appears to more than compensate for the lack of power in the critical band.
More direct evidence of the influence of nonperipheral factors was obtained from a small set of conditions in which signal thresholds were measured using the same masker for the two intervals of a single trial but different maskers across trials. This is the approach used in most previous studies of masker uncertainty in simultaneous masking with tonal complexes (e.g., Spiegel et al., 1981). Representative conditions were chosen from the peaks of the masking functions for the $1000-$ and $4000-\mathrm{Hz}$ signals for which at least $50 \mathrm{~dB}$ of masking had been observed. Ten 100-trial blocks were run, so listeners heard several hundred different maskers, but the same masker in both intervals of a given trial. To be sure that any differences observed could not be attributed to learning, thresholds for the random conditions were measured in the middle and at the end of the fixed conditions for 10 additional 100-trial runs, and these values were used for comparisons. Performance in the random conditions for both signal frequencies remained the same as in the earlier conditions for all the listeners.

Table 1 shows the amounts of masking for the 1000or $4000-\mathrm{Hz}$ signals in the presence of maskers that were either randomly drawn on each interval of each trial or fixed across the two intervals. Again, the data are averages and standard deviations across the three listeners. There is a considerable effect of fixing versus randomizing the masker frequencies across the two intervals. For the $1000-\mathrm{Hz}$ signal, performance is improved by $22 \mathrm{~dB}$ for 10-component maskers and by $13 \mathrm{~dB}$ for 20 -component maskers. Results for the $4000-\mathrm{Hz}$ signal are more variable, but in the same direction. Performance is improved by $8 \mathrm{~dB}$ for the 10-component maskers and by $25 \mathrm{~dB}$ for the 20-component maskers when the maskers are fixed across intervals. The large variability in the 20 component condition occurs because the thresholds for one listener dropped to within $10 \mathrm{~dB}$ of quiet threshold.

Clearly, it is the randomization of masker frequencies within a trial that is primarily responsible for degrading performance, despite the fact that the signal frequency is known. The fact that performance improves substantially when the same masker is used for the two listening intervals is consistent with previous work by Green and colleagues on profile analysis. That is, the listener appears to detect the signal on the basis of a qualitative change

Table 1

Average Amount of Masking in Decibels (dB) and Standard Deviations (SD) for Maskers That Were Either Fixed or Randomly Drawn for Each of the Two Intervals in a Trial

\begin{tabular}{|c|c|c|c|c|c|}
\hline \multirow{3}{*}{$\begin{array}{c}\text { Signal } \\
\text { Frequency }\end{array}$} & \multirow{3}{*}{$\begin{array}{c}\text { Masker } \\
\text { Condition }\end{array}$} & \multicolumn{4}{|c|}{ Number of Masker Components } \\
\hline & & \multicolumn{2}{|c|}{10 Tones } & \multicolumn{2}{|c|}{20 Tones } \\
\hline & & $\mathbf{d B}$ & $S D$ & $\mathbf{d B}$ & $S D$ \\
\hline \multirow[t]{2}{*}{$1000 \mathrm{~Hz}$} & Random & 55 & 7 & 53 & 8 \\
\hline & Fixed & 33 & 4 & 40 & 5 \\
\hline \multirow[t]{2}{*}{$4000 \mathrm{~Hz}$} & Random & 55 & 3 & 50 & 2 \\
\hline & Fixed & 47 & 4 & 25 & 17 \\
\hline
\end{tabular}


in the character of the masker. If large changes occur in the quality of the sound from one interval to the next, as is especially true for maskers with few components, the listener is prevented from making effective comparisons of the stimuli and large amounts of masking are produced.

Work by Spiegel et al. (1981) indicates that some masking should be produced by randomizing the masker from trial to trial, even when the masker waveforms are fixed across the observation intervals within a trial. Their maskers were multicomponent tonal complexes similar to those used in the present study, except that the masker frequencies were drawn from a $300-3000-\mathrm{Hz}$ range and the components were of equal amplitude. The effect of masker uncertainty versus signal-frequency uncertainty was examined as a function of the number of components in the maskers. They found that masker uncertainty was more disruptive than signal uncertainty regardless of the number of components in the masker, which varied from 1 to 20 . With regard to the present experiments, it is of particular interest to note that when Spiegel et al. (1981) increased the number of components from 1 to 2 , signal uncertainty increased threshold by less than $1 \mathrm{~dB}$ whereas masker uncertainty increased threshold by $6 \mathrm{~dB}$. As they pointed out, this means that monitoring two frequencies that are known is easier than monitoring only one frequency in the presence of a second tone of unknown frequency. When maskers are randomized within a trial, as they were in the present experiments, this effect is greatly enhanced.

There are two procedural issues that are important to note. The first is the issue of listener training and the second concerns the use of an adaptive procedure. With regard to training, our data show performance averaged over 10 100-trial blocks (1,000 trials) per condition. Work by Watson and his colleagues on sequential 10-tone patterns suggests that data such as these might well be subject to large training effects if listeners were practiced over many more trials (Watson, 1980). Indeed, although the thresholds were fairly stable over time, occasional sudden improvements in performance were observed. Furthermore, if detection strategies differed across conditions, there might well be differences in the time course of practice effects. In Table 1, for example, similar amounts of masking are observed for all the random conditions, but a range of masking is observed for the fixed conditions. This heterogeneity, plus the low thresholds observed on occasion for one listener, suggests that performance might well be improved with further practice in the fixed conditions: We believe that even after extensive practice, however, differences in performance for maskers randomized or fixed across the intervals would remain.

With regard to the adaptive procedure, there is the possibility that individual masker samples, drawn at random from the waveform file, could differ significantly in their ability to mask the signal. In this case, however, the adaptive procedure would act as an averager, smoothing out the variability in responses from masker to masker. Note that this is the same problem, only more exaggerated, en- countered in studies of masking with broadband noise in which individual noise samples also differ in their effectiveness as maskers. Although complete psychometric functions need to be examined in future studies, the adaptive procedure should adequately capture the differences across conditions.

Overall, several aspects of the data suggest a predominant contribution of nonperipheral factors to the large amounts of masking observed for maskers with few components. Additional peripheral factors or the interaction of multiple factors probably contribute to the sum, but these effects appear to be small in relation to those produced by masker uncertainty. The data challenge traditional models of signal detection in that listeners apparently cannot use a filter centered on the signal frequency to screen out extraneous input even if their performance could be improved by doing so.

\section{EXPERIMENT 2}

One way to gain information about how quality changes in the masker might influence performance is to vary the number of components in the masker and determine how well listeners can discriminate different numbers of components. This is similar in some respects to the experiments cited earlier that examined the ability to resolve or count components in complex spectra (Hartmann et al., 1986; Pollack, 1964; Thurlow \& Rawlings, 1959). Our primary goal was not to test the limits of spectral resolution via numerosity judgments, but to assess whether the discrimination of the number of components was a potential cue for signal detection in our masking experiment. Rather than attempt to detect a signal of a certain frequency, listeners might try simply to pick the interval with more components. This certainly would seem to be an effective strategy for maskers with small numbers of components. Experiment 2 investigated how well listeners are able to make such judgments.

\section{Method}

Three different normal-hearing listeners participated in this experiment. Two had had extensive experience with multicomponent maskers in experiments on profile analysis. The stimuli had the properties of the masker waveforms used in the previous experiment. As before, each stimulus was drawn at random from a set of 50 waveforms with a specified number of components, and was calibrated to $60 \mathrm{~dB}$ SPL average total power, with variation around that value. Thus, changes in overall power across waveforms with different numbers of components could not serve as a cue.

On each trial of a $2 \mathrm{AFC}$ nonadaptive procedure, two stimuli with different numbers of components were presented and the listeners were asked to pick the interval that contained the stimulus with the greater number. Judgments were obtained in 50-trial blocks; each block used only one combination of the base and comparison numbers summarized in Table 2 . Six blocks were run for each combination ( 300 trials per listener); the results will be presented as averages and standard deviations across listeners. To compare performance across the different bases, percent correct is plotted in terms of the ratio of base plus added components $(N+M)$ to the base $(N)$. Table 2 provides the corresponding number of components for these ratios. 
Table 2

Total Number of Components in the Comparison Interval Indicated by the Ratios Used in the Abscissa in Figure 4

\begin{tabular}{ccccccc}
\hline & \multicolumn{6}{c}{ Base Number of Components } \\
\cline { 2 - 7 }$(N+M) / N$ & 1 & 2 & 3 & 6 & 12 & 100 \\
\hline 1.34 & $*$ & $*$ & 4 & 8 & 16 & 134 \\
1.50 & $*$ & 3 & $*$ & $*$ & $*$ & $*$ \\
1.67 & $*$ & $*$ & 5 & 10 & 20 & 167 \\
2.00 & 2 & 4 & 6 & 12 & 24 & 200 \\
2.3 & $*$ & $*$ & 7 & 14 & 28 & 234 \\
3.00 & 3 & $*$ & $*$ & $*$ & $*$ & $*$ \\
\hline
\end{tabular}

Note-Asterisks indicate the condition was not run for a particular base number of components.

\section{Results and Discussion}

Figure 4 presents the percentage of correct judgments of waveforms with the greater numbers of components as a function of the ratio of the greater number to the base number of components. Performance across listeners was so similar that in most cases the error bars are smaller than the plotted symbols. The functions overlap regardless of whether the base number of components is 1 or 100 , indicating that the proportion of correct judgments is determined by the ratio of the number of components. The result is consistent with Weber's law, although the Weber fraction is certainly one of the largest found in sensory systems. In agreement with the early work of Thurlow and Rawlings (1959), listeners were remarkably poor at detecting a change in the number of components in the complex. An approximate $50 \%$ change relative to the base number is needed to detect the increase, if threshold is defined as $70.7 \%$ correct.

It seems likely that the detection of a change in the number of components is mediated by different processes as one varies the initial number of components. The change from 1 to 2 components undoubtedly depends on the detection of energy at the separate component frequencies. For larger base numbers, say from 100 to 150 components, performance may well depend on more global judgments of differences in timbre or apparent spectral density or spacing. Despite possible changes in modes of processing, however, the psychometric functions are remarkably similar for all values of the base number of components.

These data indicate that in nearly all conditions, the listeners probably could not pick the signal interval in the previous masking experiment simply by judging the number of components. The results in Figure 4 show that 2 versus 1 component $(N+M / N=2)$ is judged correctly $85 \%-90 \%$ of the time. However, $20 \mathrm{~dB}$ of masking was observed for a $4000-\mathrm{Hz}$ signal added to a 1-component masker in the earlier experiment. This may indicate that it is more difficult to make numerosity judgments when the components differ greatly in level, as would be the case when the second "component"' was the signal near threshold. Three versus 2 components $(N+M / N=1.5)$ are correctly distinguished $73 \%$ of the time, which is near the percentage correct tracked by the adaptive procedure in Experiment 1. The percentage correct for 4 versus 3 components is $64 \%$; for 8 versus 6 , it is $61 \%$ (7 vs. 6 was not tested); and for 134 versus 100 , it falls to $63 \%$. These percentages are too low to affect detection performance in the masking experiment. Instead, listeners must try to determine whether energy is present at the signal frequency.

\section{SUMMARY AND CONCLUSIONS}

This paper reports the results of two experiments that examined the processes involved in detecting a sinusoidal signal in the presence of multicomponent simultaneous maskers when different maskers are presented in each observation interval within a trial. The unexpected observation of large amounts of masking for maskers with very small numbers of components indicates that listeners are not behaving as traditional models of signal detection propose. For these conditions, little or no masker energy falls within a critical band around the signal frequency, yet as much as $55 \mathrm{~dB}$ of masking is measured. There is little evidence of improvement with training over as much as 1,000 trials, despite the fact that the signal frequency was fixed and multiple blocks of trials were completed at one frequency before another was tested. Both component frequency and amplitude vary in these maskers, and future work will determine the relative contribution of each of these factors to the masking observed. It is expected that frequency is the primary determinant of performance, however, because fixing frequency across intervals dramatically improved performance in the representative conditions tested. The latter result is consistent with work on profile analysis by Green and his colleagues, in that listeners are apparently able to compare waveforms across

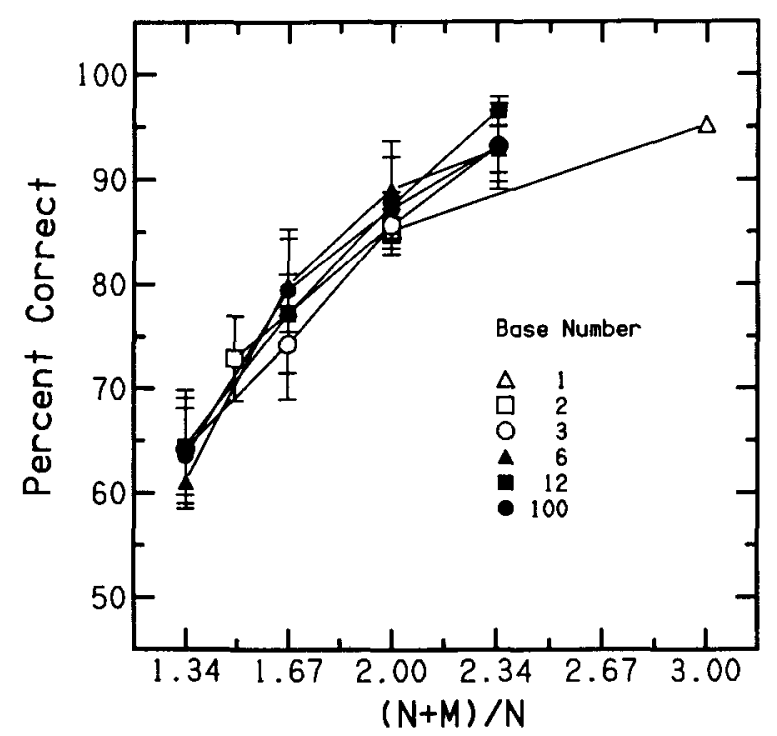

Figure 4. Percentage of correct judgments of the interval with the greater number of components. The abscissa is the ratio of the base $(N)$ plus added components $(M)$ to the base number of components. The data are averages and standard deviations across three listeners. Each point is based on six 50-trial runs per listener. 
the two intervals to detect the presence of the signal, whereas they are prevented from doing so when the maskers change with every presentation. The decrease in masking for fixed conditions is also the most direct evidence for the contribution of nonperipheral factors to the masking observed in the random conditions.

A second experiment established that listeners would have difficulty choosing the signal interval simply by judging which interval had more components. Although listeners could consistently judge proportional increases in components independent of the base number of components, performance was not good enough to maintain $70.7 \%$ correct for the detection of one additional component (the signal) in the adaptive masking task, particularly with a variable signal level. Listeners must therefore rely on some aspect of the distribution of signal and masker energy across frequency, although they apparently cannot make efficient use of a single detection filter centered at the signal frequency.

\section{REFERENCES}

Fletcher, H. (1940). Auditory patterns. Review of Modern Physics, $12,47-65$.

Green, D. M. (1983). Profile analysis: A different view of auditory intensity discrimination. American Psychologist, 38, 133-142.

GreEN, D. M., \& KIDD, G., JR. (1983). Further studies of auditory profile analysis. Journal of the Acoustical Society of America, 73, 1260-1265.

Green, D. M., Mason, C. R., \& Kidd, G., JR. (1984). Profile analysis: Critical bands and duration. Journal of the Acoustical Society of America, 75, 1163-1167.

Green, D. M., MCKeY, M. J., \& Licklider, J. C. R. (1959). Detection of a puised sinusoid in noise as a function of frequency. Journal of the Acoustical Society of America, 31, 1446-1452.

HALL, J. W. (1985). Comodulation masking release in notched noise. Journal of the Acoustical Society of America, 77 (Suppl. 1), S63.

Hall, J. W., Haggard, M. P., \& Fernandes, M. A. (1984). Detection in noise by spectrotemporal pattern analysis. Journal of the Acoustical Society of America, 76, 50-56.

Hartmann, W. M., Mcadams, S., Gerzso, A., \& Boulez, P. (1986). Discrimination of spectral density. Journal of the Acoustical Society of America, 79, 1915-1925.

LEVITT, H. (1971). Transformed up-down methods in psychoacoustics. Journal of the Acoustical Society of America, 49, 467-477.

Margolis, R. H., Dubno, J. R., \& HunT, S. M. J. (1981). Detection of tones in band-reject noise. Joumal of Speech \& Hearing Research, 24, 336-344.

NeFF, D. L., \& JesteAdT, W. (1983). Additivity of forward masking. Journal of the Acoustical Society of America, 74, 1695-1701.
Papoulis, A. (1965). Probability, random variables, and stochastic processes (pp. 461-465). New York: McGraw-Hill.

Pollack, I. (1964). Ohm's acoustical low and short-term auditory memory. Journal of the Acoustical Society of America, 36, 2340-2345.

RICHARDS, W. (1979). Quantifying sensory channels: Generalizing colorimetry to orientation and texture, touch, and tones. Sensory Processes, 3, 207-229.

Schafer, T, H., Gales, R. S., Shewmaker, C. A., \& Thompson, P. O. (1950). The frequency selectivity of the ear as determined by masking experiments. Journal of the Acoustical Society of America, 22, 490-496.

SCHARF, B. (1970). Critical bands. In J. V. Tobias (Ed.), Foundations of modern auditory theory (Vol. 1, pp. 159-202). New York: Academic Press.

SPIEGEL, M. F., \& GREEN, D. M. (1982). Signal and masker uncertainty with noise maskers of varying duration, bandwidth, and center frequency. Journal of the Acoustical Society of America, 71, 1204-1210.

Spiegel, M. F., Picardi, M. C., \& Green, D. M. (1981). Signal and masker uncertainty in intensity discrimination. Joumal of the Acoustical Society of America, 70, 1015-1019.

ThuRLOW, W. R., \& RAwLINGS, I. L. (1959). Discrimination of number of simultaneously sounding tones. Journal of the Acoustical Society of America, 31, 1332-1336.

Watson, C. S. (1976). Factors in the discrimination of word-length auditory patterns. In S. K. Hirsh, D. H. Eldredge, I. J. Hirsh, \& S. R. Silverman (Eds.), Hearing and Davis: Essays honoring Hallowell Davis (pp. 175-189). St. Louis, MO: Washington University Press.

WAtson, C. S. (1980). Time course of auditory perceptual learning. Annals of Otology, Rhinology, \& Laryngology, 89(Suppl. 74), 96-102.

WATSON, C. S., \& KELLY, W. J. (1981). The role of stimulus uncertainty in the discrimination of auditory patterns. In D. J. Getty \& J. H. Howard, Jr. (Eds.) Auditory and visual pattern recognition (pp. 37-59). Hillsdale, NJ: Erlbaum.

Watson, C. S., Kelly, W. J., \& Wroton, H. W. (1976). Factors in the discrimination of tonal patterns: II. Selective attention and learning under various levels of stimulus uncertainty. Journal of the Acoustical Society of America, 60, 1176-1186.

Weber, D. L. (1984). Combined effect of two suppressors. Journal of the Acoustical Society of America, 75, 1563-1569.

\section{NOTE}

1. Masked thresholds in the broadband-noise conditions, averaged across the three subjects, are 39.6, 40.2, and 38.5 dB SPL for the 250-, $1000-$, and $4000-\mathrm{Hz}$ signals, respectively. Corresponding predicted thresholds, based on Green, McKey, and Licklider (1959), for these signal frequencies are $38.5,40.0$, and $46.0 \mathrm{~dB}$ SPL. Thus, our listeners performed as well as, or better than, predicted in a more traditional masking condition. High thresholds in other conditions cannot be attributed simply to generally poor performance.

(Manuscript received June 20, 1986; revision accepted for publication January 23,1987 ). 